\title{
Reduced thermal conductivity of TiNiSn/HfNiSn superlattices
}

\author{
Paulina Hołuj, ${ }^{1,2, *}$ Christoph Euler, ${ }^{1}$ Benjamin Balke, ${ }^{3}$ Ute Kolb, ${ }^{4}$ Gregor Fiedler, ${ }^{5}$ Mathis M. Müller, ${ }^{6}$ Tino Jaeger, ${ }^{1}$ \\ Emigdio Chávez Angel, ${ }^{1}$ Peter Kratzer, ${ }^{5}$ and Gerhard Jakob ${ }^{1,2}$ \\ ${ }^{1}$ Institute of Physics, University of Mainz, Staudinger Weg 7, 55128 Mainz, Germany \\ ${ }^{2}$ Graduate School Materials Science in Mainz, Staudinger Weg 9, 55128 Mainz, Germany \\ ${ }^{3}$ Institute of Inorganic and Analytical Chemistry, University of Mainz, Duesbergweg 10-14, 55128 Mainz, Germany \\ ${ }^{4}$ Institute for Physical Chemistry, University of Mainz, Welderweg 11, 55099 Mainz, Germany \\ ${ }^{5}$ Faculty of Physics, University of Duisburg-Essen, Lotharstraße 1, 47048 Duisburg, Germany \\ ${ }^{6}$ Institute of Applied Geosciences, TU Darmstadt, Schnittspahnstraße 9, 64287 Darmstadt, Germany
}

(Received 15 April 2015; published 25 September 2015)

\begin{abstract}
Diminution of the thermal conductivity is a crucial aspect in thermoelectric research. We report a systematic and significant reduction of the cross-plane thermal conductivity in a model system consisting of dc sputtered TiNiSn and HfNiSn half-Heusler superlattices. The reduction of $\kappa$ is measured by the $3 \omega$ method and originates from phonon scattering at the internal interfaces. Heat transport in the superlattices is calculated based on Boltzmann transport theory, including a diffusive mismatch model for the phonons at the internal interfaces. Down to a superlattice periodicity of $3 \mathrm{~nm}$ the phonon spectrum mismatch between the superlattice components quantitatively explains the reduction of $\kappa$. For very thin individual layers the interface model breaks down and the artificial crystal shows an enhanced $\kappa$.
\end{abstract}

DOI: 10.1103/PhysRevB.92.125436

\section{INTRODUCTION}

The ability to convert a temperature difference to electricity and the possibility of both heating and cooling are very valuable properties of thermoelectric materials. Despite these promising features they are not widely used in industry as they posses low efficiency, described by a dimensionless figure of merit $Z T=\frac{S^{2} \sigma}{\kappa_{\text {ito }}} T$ ( $S$ is the Seebeck coefficient, $\sigma$ is the electrical conductivity, $\kappa_{\text {tot }}$ is the thermal conductivity, and $T$ is the absolute temperature).

$M \mathrm{NiSn}$ half-Heusler (HH) materials, where $M=\mathrm{Ti}$, Hf, and $\mathrm{Zr}$, are considered to be materials having high potential for thermoelectric (TE) applications due to Seebeck coefficients in the range of $-200 \mu \mathrm{V} / \mathrm{K}$ for bulk materials already at room temperature [1]. However, simultaneously they exhibit thermal conductivities even as high as $10 \mathrm{Wm}^{-1} \mathrm{~K}^{-1}$ preventing the achievement of a satisfactory value of $Z T$, which is a measure of the material's applicability [1]. Nowadays the scientific community is exploring all means to reduce the thermal conductivity of $\mathrm{HH}$ materials in order to enhance their $Z T$. Promising approaches include the introduction of grain boundaries by melt spinning or ball milling processes and further spark plasma sintering [2], phase separation during the solidification of bulk materials [3-6], or the thin film and superlattice (SL) approach $[7,8]$. The latter was investigated by Venkatasubramanian et al. for the $\mathrm{Bi}_{2} \mathrm{Te}_{3} / \mathrm{Sb}_{2} \mathrm{Te}_{3} \mathrm{SL}$ system and resulted in the highest ever reported $Z T$ value of 2.4 at room temperature $[9,10]$. In contrast to tellurides, $\mathrm{HH}$ materials possess the peak of efficiency at high temperature (above

*holuj@uni-mainz.de

Published by the American Physical Society under the terms of the Creative Commons Attribution 3.0 License. Further distribution of this work must maintain attribution to the author(s) and the published article's title, journal citation, and DOI.
PACS number(s): 44.10.+i, 81.15.Cd, 84.60.Rb, 68.65.Cd

$700 \mathrm{~K})$ [11]. Moreover, the cost of bulk HHs is about 1 order of magnitude lower compared to the former compounds [12].

The superlattice approach is a promising way to reduce the thermal conductivity; however, the total thermal conductivity consists of an electronic and a phononic contribution $\left(\kappa_{\mathrm{tot}}=\kappa_{\mathrm{el}}+\kappa_{\mathrm{ph}}\right)$. The first is directly related to the electrical conductivity via the Wiedemann-Franz law $\left(\frac{\kappa_{\mathrm{el}}}{\sigma T}=\right.$ const $)$ [13]. Therefore, reduction of $\kappa_{\mathrm{el}}$ leads to a reduction of $\sigma$ and to a decrease of $Z T$. To ensure that $\kappa_{\mathrm{el}}$ and other electronic properties remain unchanged, isoelectronic elements are used as substitutes for the $M$ element in the compounds so that mainly the phononic thermal conductivity is affected. In our model system SLs have constant total thicknesses, but a variable SL period and therefore a variable number of interfaces. However, SLs with ultrashort period consist only of a few atomic planes of the different materials. Thus they should better be considered not as layers of material 1 and material 2 separated by interfaces, but rather as an artificial tailor-made new material with a large crystallographic unit cell.

\section{EXPERIMENTAL DETAILS}

TiNiSn/HfNiSn SLs having the same amount of both materials in one period were grown by dc magnetron sputtering. Details about sample preparation are presented in the Supplemental Material [14]. The cross-plane thermal conductivity of HH SLs was measured with the $3 \omega$ method [15-17]. The sample geometry and the measurement procedure are described in detail in our previous work [7] and in the Supplemental Materials [14].

\section{RESULTS AND DISCUSSION}

\section{A. Structure of the superlattices}

A crucial aspect of the approach pursued here is a strong sensitivity to the quality of the interfaces. X-ray diffraction (XRD), atomic force microscopy (AFM), and scanning transmission electron microscopy (STEM) were used 

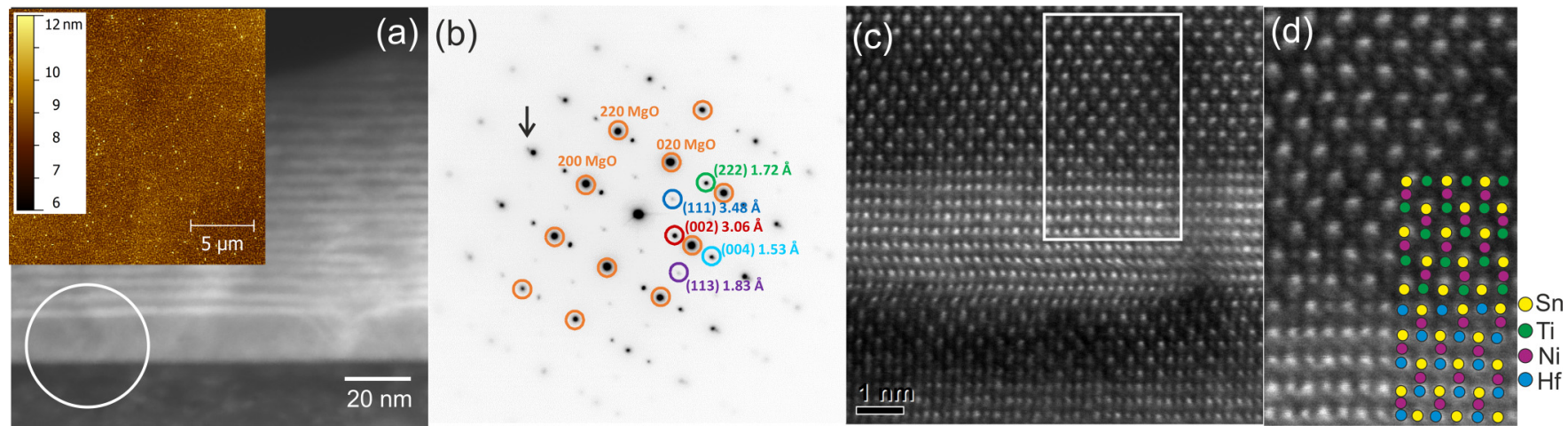

FIG. 1. (Color online) (a) STEM dark field image of a SL cross section with a period of $4 \mathrm{~nm}$, composed of TiNiSn (dark contrast) and HfNiSn (bright contrast) and surface topography of an approximately 800-nm-thick SL with rms roughness of $1.1 \mathrm{~nm}$ (AFM image) in the inset. (b) SAED of a circular region from the image in panel (a). (c) High-resolution STEM image. (d) The magnification of a rectangular region with the assignment of atoms.

to estimate interface quality. Assuming that the interface roughness transfers to the surface roughness, a root mean square (rms) roughness of $1.1 \mathrm{~nm}$ for an 800 -nm-thick SL [Fig. 1(a), inset] implies a smooth boundary between layers. Moreover, the STEM image shown in Fig. 1(a) proves the ability to grow high-quality structures. In this particular case, a SL with a period of about $4 \mathrm{~nm}$ is presented. Every bright layer of the stack corresponds to HfNiSn, whereas dark layers demonstrate the presence of TiNiSn.

Additionally, the selected area electron diffraction (SAED) of a circular region marked in Fig. 1(a) was measured and the result, together with the assignment of crystalline directions to the reflections, is presented in Fig. 1(b). Every orange circle indicates a $\mathrm{MgO}$ diffraction spot, whereas other colors refer to film reflections. Observed diffraction spots confirm strongly directional growth of the film according to the orientation provided by the substrate. Due to the slight difference between lattice constants of both $\mathrm{HH}$ materials, we also observe a splitting of the reflection indicated by an arrow. Unequal lattice constants lead to the distortion of the lattice visible in the high-resolution STEM image [Fig. 1(c)]. Based on the brightness contrast, one can clearly recognize piles of the constituent atoms.

While AFM and STEM yield local information, XRD averages over a large area and is sensitive to inner interfaces, since the films are thinner than the penetration depth. Typical XRD data are shown in Fig. 2. The [001] ([100]) direction of the film is parallel to the [001] ([110]) direction of the $\mathrm{MgO}$ substrate as is evident by the occurrence of (002) and (004) film peaks. Additional satellites around the main diffraction lines of TiNiSn and HfNiSn arise from the extra periodicity present in the samples [18]. These satellite peaks are particularly sensitive to the quality of the boundaries between the constituent layers as any continuous fluctuations would cause broadening of all SL peaks resulting in difficulties in resolving additional oscillations [18]. Consequently, pronounced satellites imply better sample quality.

In Fig. 2, besides measured XRD data, the calculation based on coherent $\mathrm{x}$-ray scattering for a perfect SL is presented. To give a realistic physical shape to the simulated spectra, peaks were convoluted with the measured profiles of epitaxial TiNiSn films. Convolution parameters resulted from the fit of two Pseudo-Voigt profiles to (002) and (004) peaks for single TiNiSn films, respectively. Scattering factors of the atoms in the planes were taken from Ref. [19] and the lattice plane spacing was adjusted to account for epitaxial strain. Therefore, instead of bulk lattice parameters of $\mathrm{HH}$ films $\left(a_{\mathrm{TiNiSn}}=5.941 \AA, a_{\mathrm{HfNiSn}}=6.083 \AA\right)$ [20], the out-of-plane lattice parameters taken for the calculation were equal to $a_{\mathrm{TiNiSn}}=5.906 \AA$ and $a_{\mathrm{HfNiSn}}=6.184 \AA$. The thicknesses of TiNiSn and HfNiSn were set to 10.6 and $11.3 \mathrm{~nm}$, respectively. Comparing measured and calculated spectra of the SLs, it can be concluded that not only the positions of the peaks but also the overall peak shapes agree well. However, relative intensities of the peaks are not exactly reproduced, especially for higher-order satellites, indicating disturbances from the perfect periodicity in the experimental samples.

To measure the cross-plane thermal conductivity the differential $3 \omega$ method was used. The $U_{3 \omega}$ in the heater structure was measured by an SR850 DSP lock-in amplifier from Stanford Research Systems. For every SL period two sets of regular and reference samples were prepared in order to confirm the reproducibility. Based on both pairs of results the error bars of

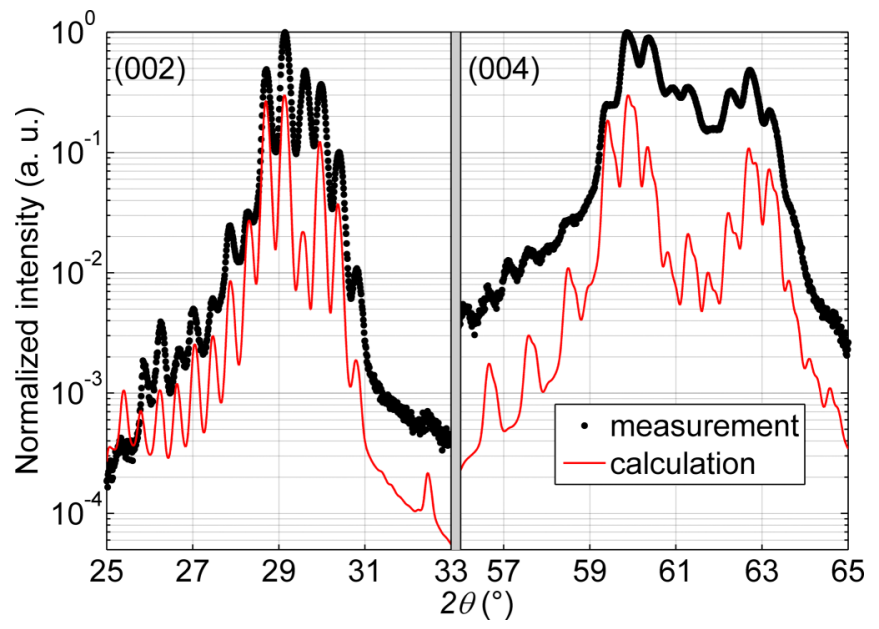

FIG. 2. (Color online) Measured and calculated XRD $\theta-2 \theta$ profile of a SL with a period of $21.9 \mathrm{~nm}$. The intensities are rescaled to create an offset for clarity. Shown are the regions around (002) and (004) reflection. 


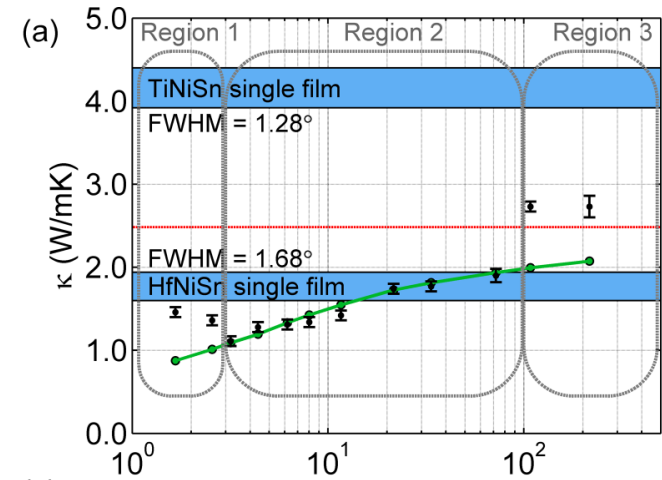

(c)

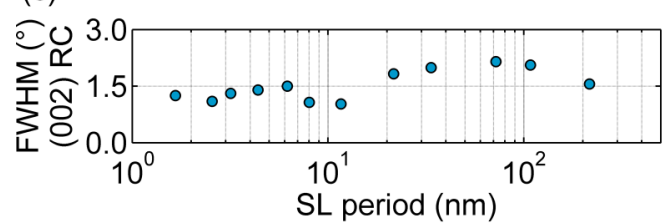

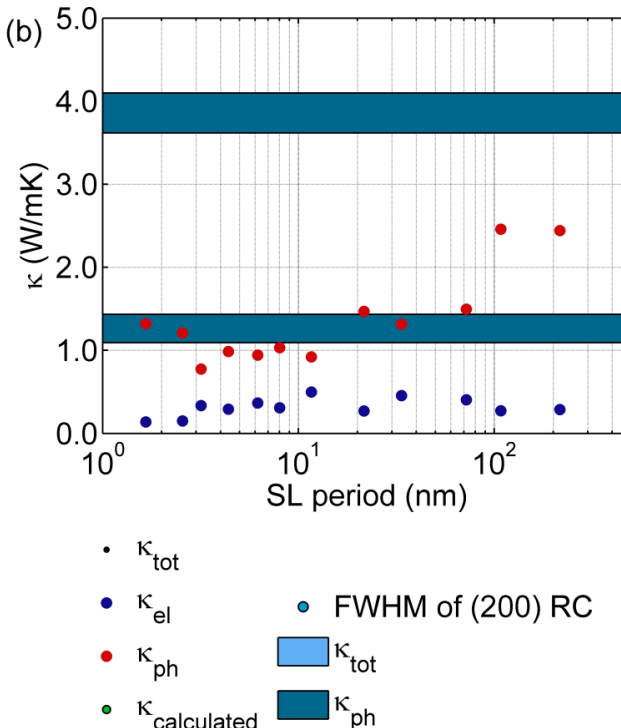

FIG. 3. (Color online) (a) Measured cross-plane thermal conductivity of HH SLs with varying period (black dots) and $\kappa_{\text {calculated }}$ based on a series of thermal resistances (green dots). Experiment and calculation agree for SL periods in region 2. (b) Electronic and phononic part of the thermal conductivity for every SL and single films. (c) FWHM of the (002) rocking curves of the films and SLs.

data points were estimated. The difference between the thermal conductivities of both series of samples having the same SL period was compared with an uncertainty originating from the measurement setup. Then the error bars were assumed to be equal to the greater of these values.

Besides the measurement of the thermal conductivity, the $\mathrm{X}$-ray diffraction rocking curves (RC) of the (002) diffraction peak or the most intense satellite were acquired for one thick sample of every studied SL period. RCs were fitted with a Pseudo-Voigt profile and their full width at half maximum (FWHM) values for every period are summarized in Fig. 3(c). Contrary to our previous study on ( $\mathrm{Zr}, \mathrm{Hf}) \mathrm{NiSn}$ /TiNiSn superlattices [7], we do not see any correlation between the width of the RCs and the thermal conductivity in the TiNiSn/HfNiSn system which indicates the improved growth of the system investigated here.

\section{B. Thermal conductivity}

The study of room-temperature thermal conductivity versus the SL period is presented in Fig. 3(a). The horizontal blue bars indicate the thermal conductivity of the individual film materials shown for comparison. For TiNiSn films we obtained an average of $4.16 \mathrm{Wm}^{-1} \mathrm{~K}^{-1}$. Due to lower roughness and improved sample crystalline alignment in the current sample series this value is almost $50 \%$ higher than the one reported by us earlier $\left(2.8 \mathrm{Wm}^{-1} \mathrm{~K}^{-1}\right)$ [7]. The current result is also much closer to the bulk value of the thermal conductivity of TiNiSn reported in the literature, which ranges between 4.6 and $9.3 \mathrm{Wm}^{-1} \mathrm{~K}^{-1}$ for arc-melted [2,21,22], $4.8 \mathrm{Wm}^{-1} \mathrm{~K}^{-1}$ for microwave-prepared [21], and $7.5 \mathrm{Wm}^{-1} \mathrm{~K}^{-1}$ for levitationmelted materials [23]. Conversely, we report a reduced thermal conductivity down to $1.77 \mathrm{Wm}^{-1} \mathrm{~K}^{-1}$ for HfNiSn thin films compared to literature data on bulk material that varies between 6.7 and $10 \mathrm{Wm}^{-1} \mathrm{~K}^{-1}[1,22]$. As the mismatch between the $\mathrm{MgO}$ substrate and HfNiSn is considerably larger than that for TiNiSn, the former shows wider rocking curves, i.e., worse crystallite alignment leading to a reduced thermal conductance. The data shown in this article, however, have been obtained on samples with comparable crystalline quality.

The black data points presented in Fig. 3(a) indicate that indeed a systematic reduction of the thermal conductivity was achieved. The wide range of studied SL periods may be divided into three regions. For large periods, labeled as region 3, we observe $\kappa_{\text {tot }}$ very close to a level marked with a red line that represents the $\kappa_{\text {series }}$ of a bilayer in which the effect of internal interfaces is ignored and the value is calculated from the arithmetic mean of the measured series heat resistances of the constituent layers,

$$
\kappa_{\text {series }}=\frac{d_{1}+d_{2}}{\frac{d_{1}}{\kappa_{\mathrm{TiNiSn}}}+\frac{d_{2}}{\kappa_{\mathrm{HfNiSn}}}},
$$

where $d_{1}$ and $d_{2}$ are the total thicknesses of TiNiSn and HfNiSn. One can also notice an apparent saturation of the thermal conductivity for high period lengths, because only a few interfaces (15 and 7 for periods equal to 108 and $216 \mathrm{~nm}$, respectively) are not significantly affecting $\kappa_{\mathrm{tot}}$. In region 2 the reduction of the thermal conductivity is much more significant and $\kappa_{\text {tot }}$ decreases systematically when the number of interfaces increases from 23 to 575. In this region, an appropriate model is the series of thermal resistances that include both the measured thermal resistivities of the individual films and the interface thermal resistance. To simulate the thermal conductivity we apply semiclassical Boltzmann transport, based on the ab initio calculation of the bulk phonons using density-functional perturbation theory [24]. To get the thermal conductivity one has to integrate the phonon spectra over the total $q$ space summing up all phonon modes $j$. As an approximation we take the phonons as isotropic so that the angular integration gives just a constant:

$$
\kappa_{\mathrm{bulk}, x}=\frac{1}{8 \pi^{2}} \sum_{j} \int_{q_{j, x}} \hbar \omega_{j, x} q_{j, x}^{2}\left|v\left(q_{j, x}\right)\right|^{2} \tau_{x} \frac{d N_{0}}{d T} d q_{j, x},
$$


where $v\left(q_{j, x}\right)$ is the group velocity of the phonon mode of the material $x$ and $N_{0}$ is the Bose distribution function. It is well established [25] that $\tau$ has the form of

$$
\tau_{x}=\frac{A_{x}}{\omega_{j, x}^{2} T} .
$$

We choose $A_{x}$ such that the above integral reproduces the experimental bulk thermal conductivity. For the interfaces the mean free path $\ell=v \tau$ must be modified by the transmission coefficient $\zeta_{x \rightarrow y}$ and the material layer thickness $d$. This is obtained by a diffusive mismatch model [26], which is based on the mismatch of the bulk phonons of the two materials. The frequency-dependent transmission through the layer and the interface is then given by

$$
\kappa_{x}=\frac{1}{8 \pi^{2}} \sum_{j} \int_{q_{j, x}} \hbar \omega_{j, x} q_{j, x}^{2}\left|v\left(q_{j, x}\right)\right| \ell\left(q_{j, x}\right) \frac{d N_{0}}{d T} d q_{j, x},
$$

with an interface and thickness-dependent mean free path,

$$
\frac{1}{\ell\left(q_{j, x}\right)}=\frac{1}{d_{x} \zeta_{x \rightarrow y}\left(q_{j, x}\right)}+\frac{\omega_{j, x}^{2} T}{A_{x}\left|v\left(q_{j, x}\right)\right|} .
$$

Equation (4) gives the thermal conductivity of material $x$ with layer thickness $d_{x}$ including the interface through which the phonon leaves the layer and is used in Eq. (1). The green circles in Fig. 3(a) that have been obtained from such modeling indicate that the interpretation of the experimental data using thermal interface resistances works for SL periods down to $3 \mathrm{~nm}$ [region 2 in Fig. 3(a)]. For thinner SLs the model is no longer valid because the long-wavelength phonons experience an effective medium formed by both materials (see below), which results in a modified phonon dispersion as compared to each individual material.

Finally, for a SL period of $3.2 \mathrm{~nm}$, the lowest thermal conductivity of $1.11 \pm 0.06 \mathrm{Wm}^{-1} \mathrm{~K}^{-1}$ was measured. Decreasing the period even more (region 1), we observed an increase of $\kappa_{\text {tot }}$ due to the formation of an artificial crystal. Along the [100] direction the crystallographic unit cell of a $\mathrm{HH}$ compound is composed of $2 \times 2=4$ atomic layers, where 2 neighboring atomic layers form the chemical unit cell. For the sample with the lowest period of $1.7 \mathrm{~nm}$ the new crystal consists of 1.25 unit cells of TiNiSn and 1.5 unit cells of HfNiSn, i.e., in total only 11 atomic layers. Locally the chemistry of the $\mathrm{HH}$ material requires the crystal size to be 10 or 12 atomic layers, whereas $\mathrm{x}$ rays give the average value. In such a system, phonons experience the material as if it was composed of enlarged unit cells. In this case the still reduced thermal conductance must be attributed to a backfolding of the phonon dispersion relation due to the supercell that introduces gaps in the phonon dispersion relation at the new Brillouin zone boundaries and leads to an overall reduced dispersion of the phonon branches [27].

The decomposition of the thermal conductivity into electronic and phononic contributions is summarized in Fig. 3(b). The electronic part of the thermal conductivity $\kappa_{\mathrm{el}}$, calculated according to the Wiedemann-Franz law, stays relatively constant for all SL periods, whereas the phononic part follows the trend appointed by $\kappa_{\text {tot }}$. Thereby, the reduction of the cross-plane thermal conductivity can be definitely assigned to the phonons scattering at the interfaces.

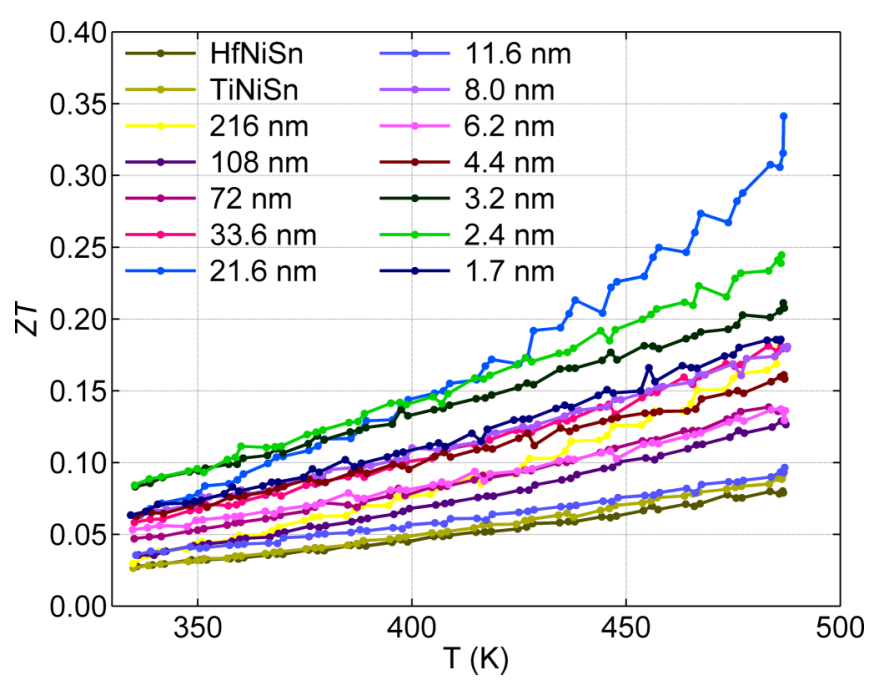

FIG. 4. (Color online) $Z T$ values calculated from in-plane Seebeck coefficient and electrical resistivity and cross-plane thermal conductivity for TiNiSn and HfNiSn and for their SLs.

\section{Power factor}

The in-plane resistivity and the Seebeck coefficient were measured simultaneously by an LSR 3 (Linseis) in a $\mathrm{He}$ atmosphere between room temperature and $480 \mathrm{~K}$. The plot summarizing the measured data is presented in the Supplemental Material [14]. Due to the isoelectronic substitution we expect a lower interface scattering for electrons than for phonons and assume to first approximation isotropic electronic transport. This assumption is corroborated by the absence of a systematic correlation between the electronic properties and the interface density. The scattering of the values of the thermopower $S$ is reflected by a corresponding but reciprocal scattering of the Hall carrier concentration (not shown here). This indicates that despite nominally equal deposition conditions there is some fluctuation of the doping level between the superlattices.

As obviously the electronic transport properties are not dominated by the anisotropy introduced with the layering, we used the room-temperature cross-plane thermal conductivity and the temperature-dependent in-plane power factor to estimate the $Z T$ values and we present them in Fig. 4. The figure of merit of both single films is almost identical along the entire temperature range and reaches a value of about 0.08 at $480 \mathrm{~K}$, whereas all the studied SLs exhibit greater $Z T$ values. In the extreme case, for a SL having a period of $21.6 \mathrm{~nm}, Z T$ reaches a value of almost 0.35 , which is 4.4 times larger than the figure of merit of single films. The currently achieved value falls well within the interval demarcated by the previously reported data $[4,6,21,28-34]$ at $480 \mathrm{~K}$.

We deduce that both approaches, top-down (doping and phase separation) as well as bottom-up (SLs), are complementary ways to enhance the figure of merit. While the former has no control over the inclusions size, the latter can vary the thickness of layers with great accuracy. As presented above, the figure of merit of 0.35 for the SL is comparable to the best $Z T$ values for bulk $\mathrm{HH}$ alloys reported in the literature at $480 \mathrm{~K}$. 


\section{CONCLUSIONS}

In conclusion, the present research was designed to study the effect of interfaces on the thermal conductivity in $\mathrm{HH}$ SLs. Direct current sputtered TiNiSn/HfNiSn SLs reveal a smooth surface and well-defined layered structure as proved by AFM, XRD, and STEM. The thermal conductivity of thin films was measured using the differential $3 \omega$ method. Decreasing the SL period, i.e., increasing the number of interfaces, the expected reduction of the thermal conductivity was observed. For a SL period of $3.2 \mathrm{~nm}$ the minimum $\kappa_{\mathrm{tot}}=1.11 \pm 0.06 \mathrm{Wm}^{-1} \mathrm{~K}^{-1}$ was achieved. Further decrease of the SL period leads to the rise of $\kappa_{\text {tot }}$ due to the creation of an artificial crystal. We calculated the thermal conductivity using semiclassical Boltzmann transport, based on the ab initio calculation of the bulk phonons using density-functional perturbation theory and a diffusive mismatch model for phonon scattering at the interfaces. Experimental data and calculation agree for SL periods larger than $3 \mathrm{~nm}$. An electron confinement might be the reason for the improved Seebeck coefficient in SLs with very low periods. The figure of merit was estimated based on the room temperature cross-plane thermal conductivity and the temperature-dependent in-plane power factor. Due to the reduced thermal conductivity, the SLs with all studied periods reveal enhanced $Z T$ compared to single constituent films.

\section{ACKNOWLEDGMENTS}

We gratefully acknowledge financial support by the Deutsche Forschungsgemeinschaft (DFG) [Grants No. Ja821/4, No. BA4171/2-2, and No. KR2057/4-2 within SPP 1386 (Nanostructured Thermoelectric Materials) and No. Ja821/7-1 within SPP 1538 (Spin Caloric Transport)] and the Graduate School of Excellence, Material Science in Mainz (GSC 266).
[1] C. Uher, J. Yang, S. Hu, D. T. Morelli, and G. P. Meisner, Phys. Rev. B 59, 8615 (1999).

[2] Y. Gelbstein, N. Tal, A. Yarmek, Y. Rosenberg, and M. P. Dariel, J. Mater. Res. 26, 1919 (2011).

[3] E. Rausch, B. Balke, S. Ouardi, and C. Felser, Phys. Chem. Chem. Phys. 16, 25258 (2014).

[4] Y. Kimura, H. Ueno, and Y. Mishima, J. Electron. Mater. 38, 934 (2009).

[5] S. Populoh, M. Aguirre, O. Brunko, K. Galazka, Y. Lu, and A. Weidenkaff, Scr. Mater. 66, 1073 (2012).

[6] M. Schwall and B. Balke, Phys. Chem. Chem. Phys. 15, 1868 (2013).

[7] T. Jaeger, P. Hołuj, C. Mix, C. Euler, M. H. Aguirre, S. Populoh, A. Weidenkaff, and G. Jakob, Semicond. Sci. Technol. 29, 124003 (2014).

[8] S.-H. Wang, H.-M. Cheng, R.-J. Wu, and W.-H. Chao, Thin Solid Films 518, 5901 (2010).

[9] R. Venkatasubramanian, Phys. Rev. B 61, 3091 (2000).

[10] R. Venkatasubramanian, E. Siivola, T. Colpitts, and B. O’Quinn, Nature (London) 413, 597 (2001).

[11] T. Graf, C. Felser, and S. S. Parkin, Prog. Solid State Chem. 39, 1 (2011).

[12] S. LeBlanc, S. K. Yee, M. L. Scullin, C. Dames, and K. E. Goodson, Renewable Sustainable Energy Rev. 32, 313 (2014).

[13] C. Kittel, Introduction to Solid State Physics (Wiley \& Sons, New York, 2008)

[14] See Supplemental Material at http://link.aps.org/supplemental/ 10.1103/PhysRevB.92.125436 for details about the sample preparation, the 3omega method, and the measured thermoelectric quantities.

[15] D. G. Cahill, Rev. Sci. Instrum. 61, 802 (1990).

[16] D. G. Cahill, Rev. Sci. Instrum. 73, 3701 (2002).

[17] T. Borca-Tasciuc, A. R. Kumar, and G. Chen, Rev. Sci. Instrum. 72, 2139 (2001).

[18] E. E. Fullerton, I. K. Schuller, H. Vanderstraeten, and Y. Bruynseraede, Phys. Rev. B 45, 9292 (1992).
[19] Edited by E. Prince, International Tables for Crystallography, Volume C, Mathematical, Physical and Chemical Tables (Kluwer Academic, Dordrecht, 2004).

[20] W. Jeitschko, Metall. Trans. 1, 3159 (1970).

[21] C. S. Birkel, W. G. Zeier, J. E. Douglas, B. R. Lettiere, C. E. Mills, G. Seward, A. Birkel, M. L. Snedaker, Y. Zhang, G. J. Snyder, T. M. Pollock, R. Seshadri, and G. D. Stucky, Chem. Mater. 24, 2558 (2012).

[22] H. Hohl, A. P. Ramirez, C. Goldmann, G. Ernst, B. Wölfing, and E. Bucher, J. Phys. Condens. Matter 11, 1697 (1999).

[23] J. E. Douglas, C. S. Birkel, M.-S. Miao, C. J. Torbet, G. D. Stucky, T. M. Pollock, and R. Seshadri, Appl. Phys. Lett. 101, 183902 (2012).

[24] S. Baroni, S. de Gironcoli, and A. D. Corso, Rev. Mod. Phys. 73, 515 (2001)

[25] P. Klemens, Proc. R. Soc., Ser. A 208, 108 (1951).

[26] T. Beechem, J. C. Duda, P. Hopkins, and P. M. Norris, Appl. Phys. Lett. 97, 061907 (2010).

[27] S. I. Tamura, Y. Tanaka, and H. J. Maris, Phys. Rev. B 60, 2627 (1999).

[28] C. S. Birkel, J. E. Douglas, B. R. Lettiere, G. Seward, N. Verma, Y. Zhang, T. M. Pollock, R. Seshadri, and G. D. Stucky, Phys. Chem. Chem. Phys. 15, 6990 (2013).

[29] K. Kurosaki, T. Maekawa, H. Muta, and S. Yamanaka, J. Alloys Compd. 397, 296 (2005).

[30] R. A. Downie, D. A. MacLaren, R. I. Smith, and J. G. Bos, Chem. Commun. 49, 4184 (2013).

[31] H. Muta, T. Kanemitsu, K. Kurosaki, and S. Yamanaka, J. Alloys Compd. 469, 50 (2009).

[32] S. J. Poon, D. Wu, S. Zhu, W. Xie, T. M. Tritt, P. Thomas, and R. Venkatasubramanian, J. Mater. Res. 26, 2795 (2011).

[33] K. Gałązka, S. Populoh, W. Xie, S. Yoon, G. Saucke, J. Hulliger, and A. Weidenkaff, J. Appl. Phys. 115, 183704 (2014).

[34] H. Geng and H. Zhang, J. Appl. Phys. 116, 033708 (2014). 